Supplemental Information:

\title{
Spinosyn G: Proof of Structure by Semi-synthesis
}

Paul R. Graupner et al.

\section{$\underline{\text { Contents }}$}

$\begin{array}{lr}\text { Contents; General Experimental Details } & \text { S1 } \\ \text { 1H NMR spectrum of natural Spinosyn G (1) } & \text { S2 } \\ \text { 1H NMR specrum of 4"-epi-spinsyn A (4) } & \text { S3 } \\ \text { 1H NMR spectrum of anomer of spinosyn G (19) } & \text { S4 } \\ \text { 1H NMR spectrum of synthetic spinosyn G (1) } & \text { S5 } \\ \text { Comparison of natural vs. synthetic 1 } & \text { S6 }\end{array}$

$\underline{\text { Numbering Scheme for Spinosyn Molecules }}$

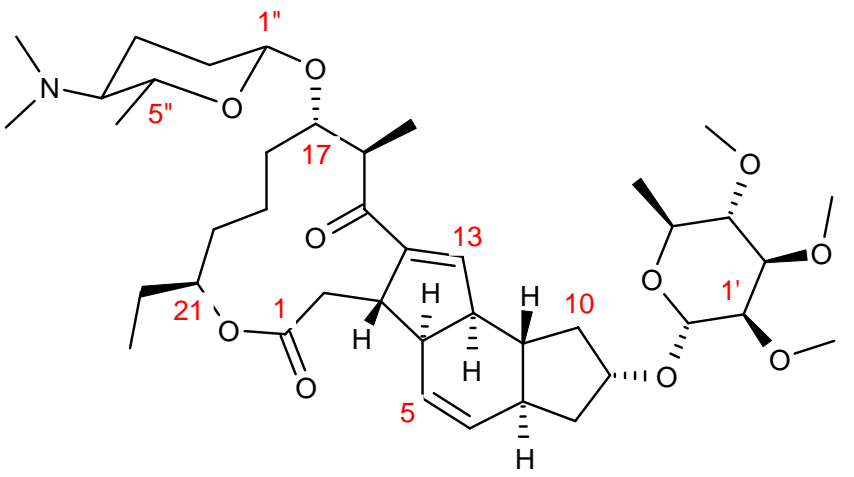

$\underline{\text { General Experimental Details }}$

General Methods. All NMR data were obtained at 600 or $400 \mathrm{MHz}$ in $\mathrm{CDCl}_{3}$, using residual solvent signals as an internal standard $\left({ }^{1} \mathrm{H}\right.$ at $7.27,{ }^{13} \mathrm{C}$ at 77.4$)$. 
$1 \mathrm{H}$ spectrum $(600 \mathrm{MHz}$, resolution enhanced) of 1 (Spinosyn G-natural product) in chloroform-d

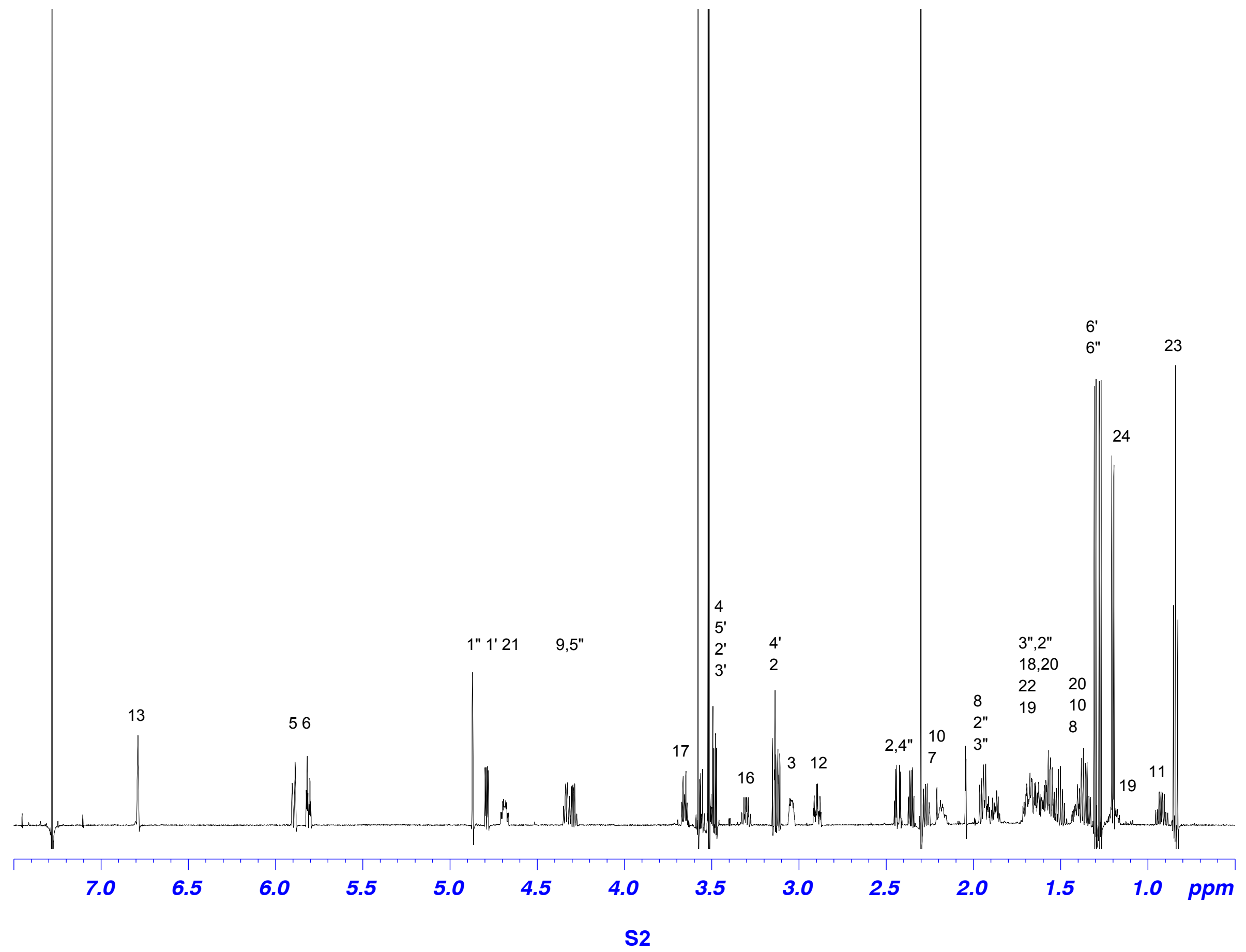


$1 \mathrm{H}$ spectrum (400MHz, resolution enhanced) of 4 (beta-D-ossaminyl spinosyn) in chloroform-d

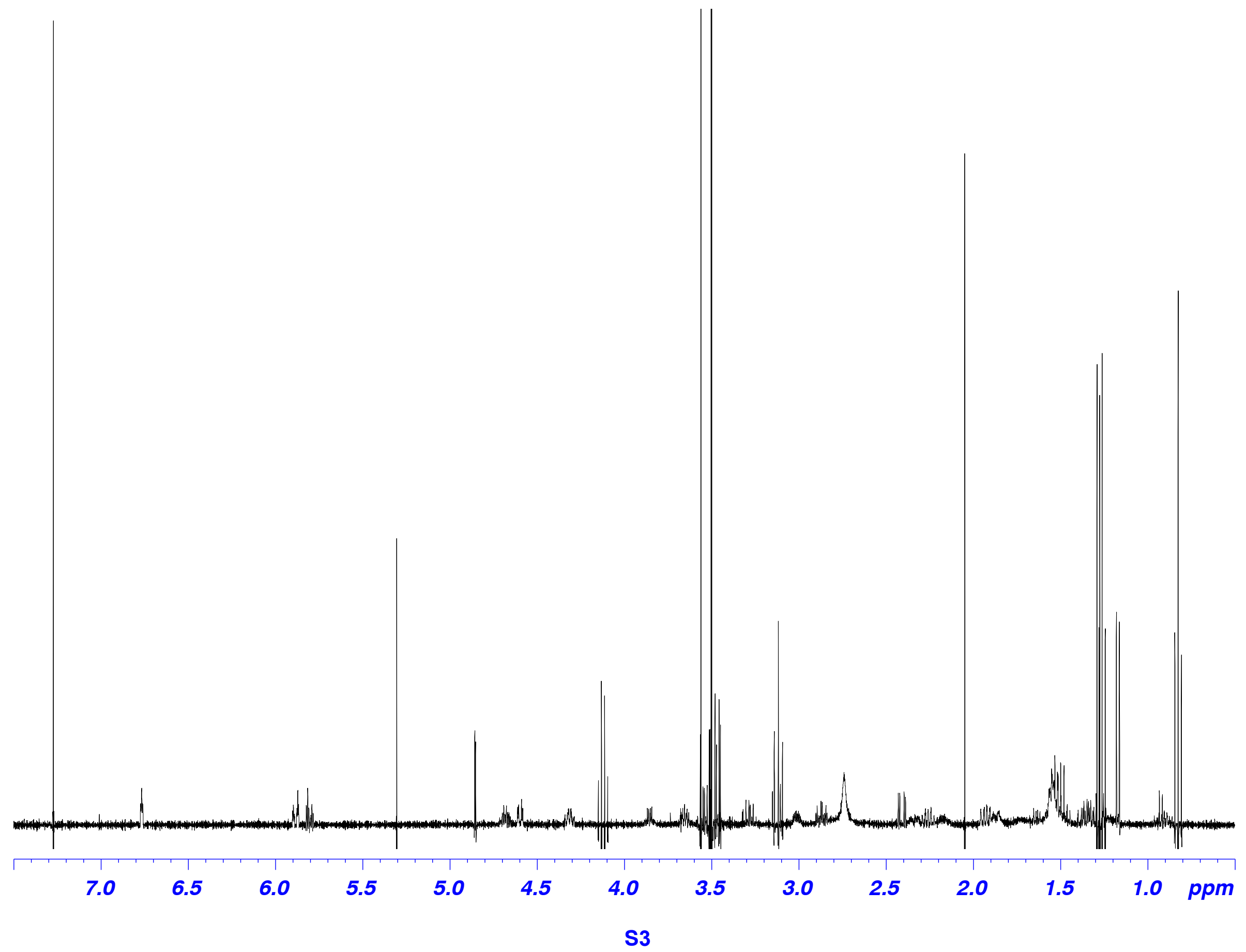


$1 \mathrm{H}$ spectrum $(600 \mathrm{MHz}$, resolution enhanced) of 19 (beta-L-ossaminyl spinosyn) in chloroform-d

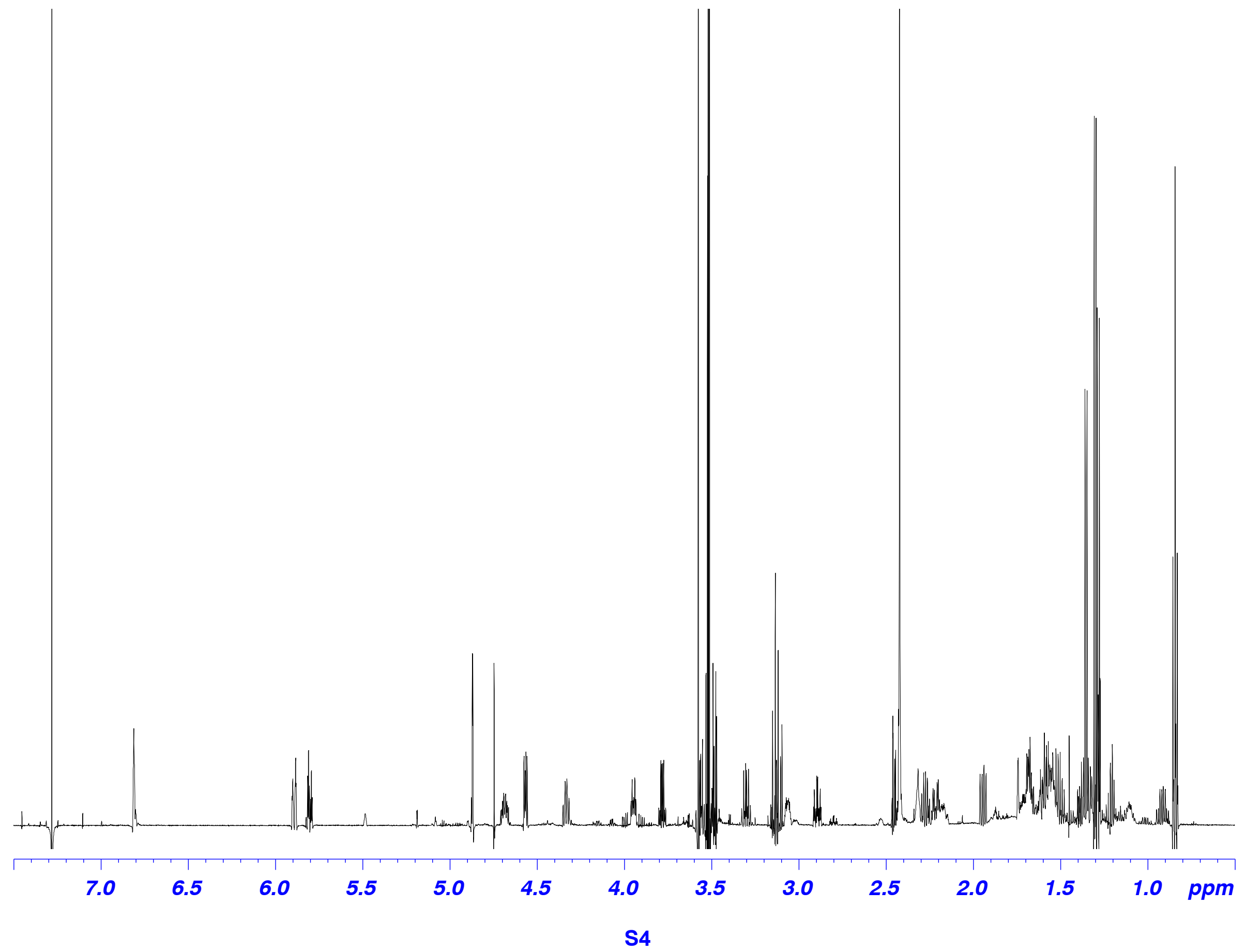


$1 \mathrm{H}$ spectrum (600MHz, resolution enhanced) of 1 (spinosyn G - semisynthetic) in chloroform-d
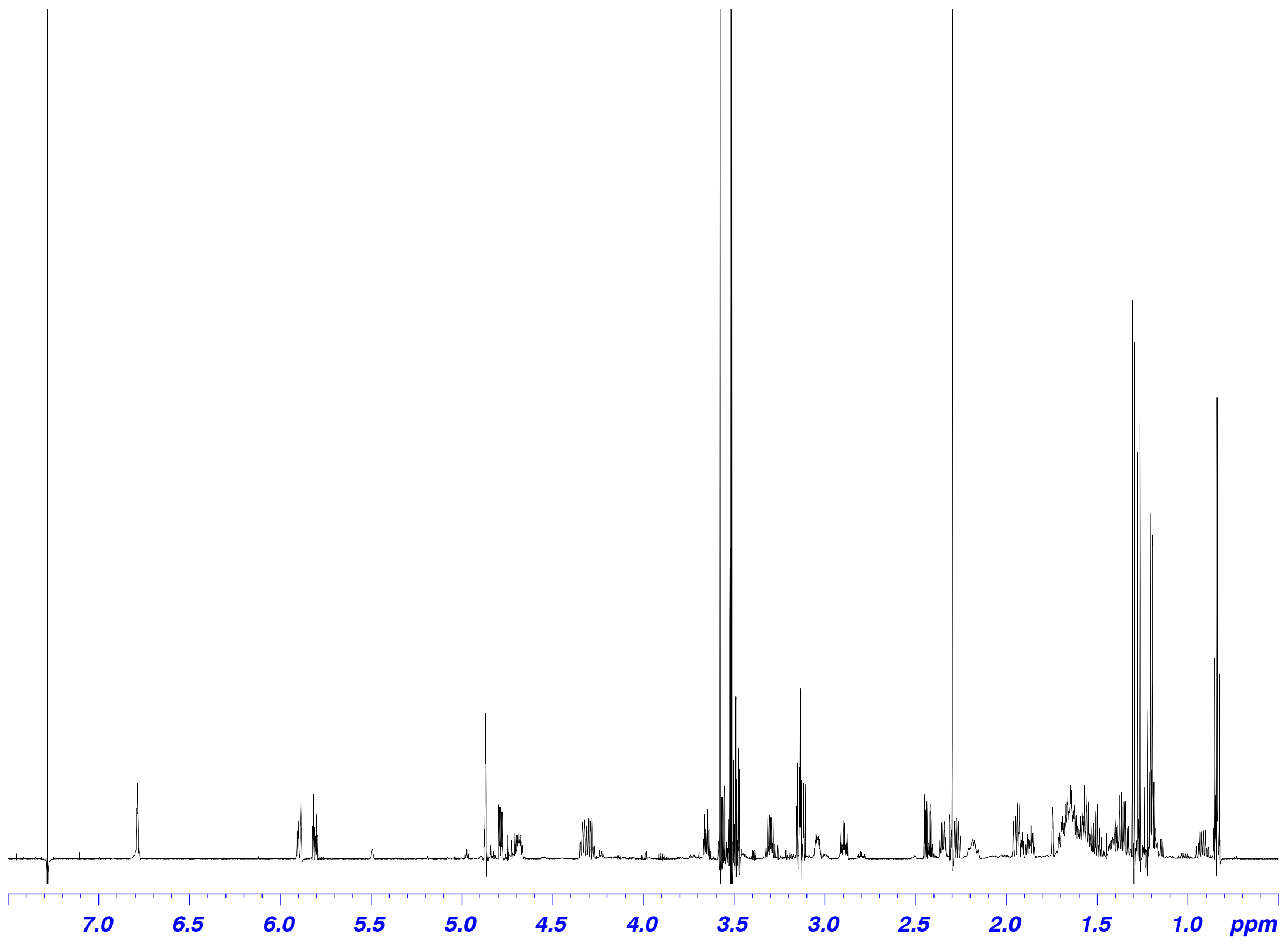

S5 
$1 \mathrm{H}$ spectrum (600MHz, resolution enhanced) of 1; Natural (bottom) and semi-synthetic (top) spinosyn G in chloroform-d
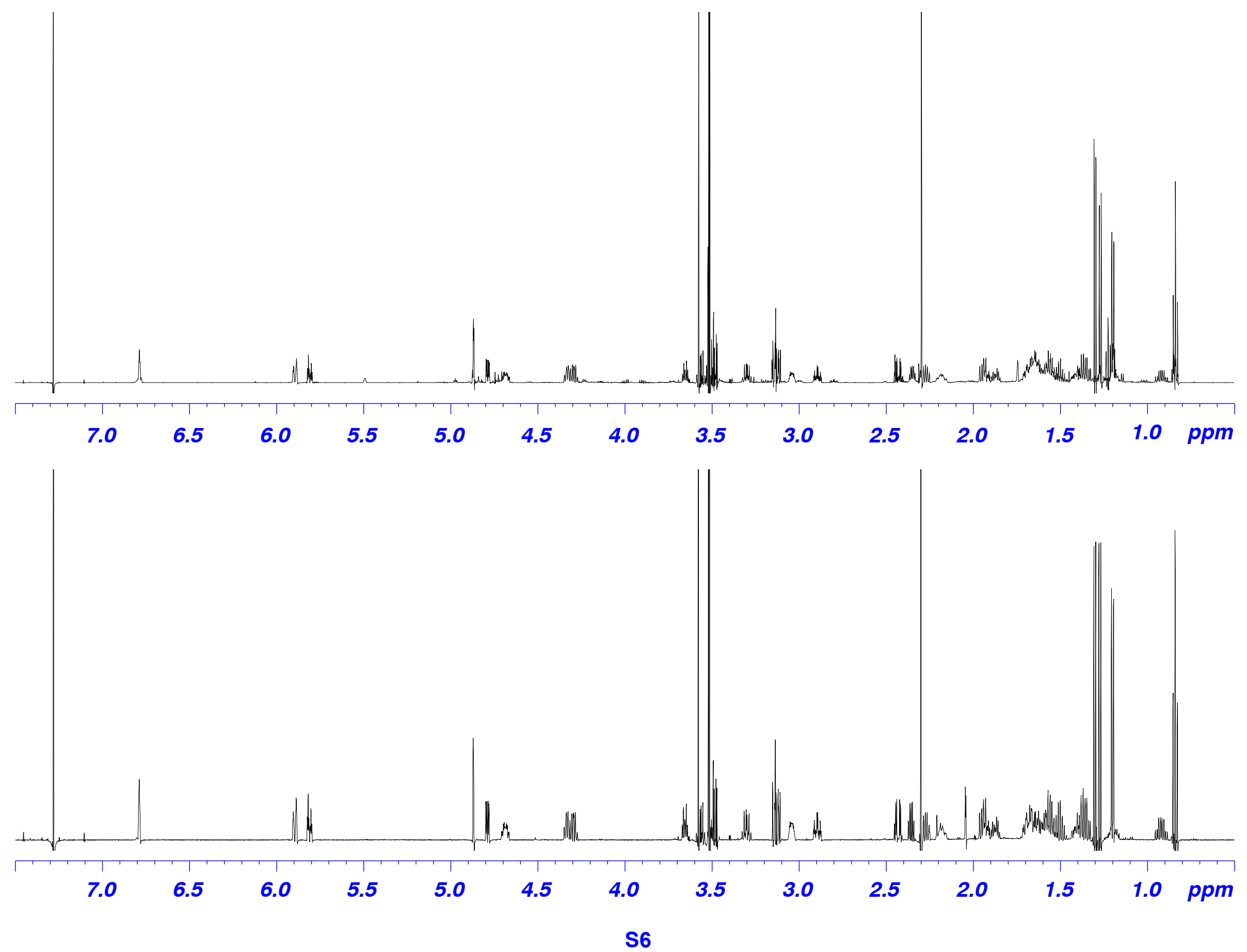